\title{
A Study on Skin Properties Related to Chronic Psychological Stress
}

\author{
Sue Im Jang, Yuri Seol, Eun Joo Kim, and Haekwang Lee*. \\ AMOREPACIFIC R\&D Unit, Gyeonggi-do, 17074, Republic of Korea \\ *haekwang@amorepacific.com
}

\section{INTRODUCTION}

These days many people live under a lot of stress in everyday life. According to many studies, chronic or long-term stress has bad effects on health. Several studies have found that the skin barrier function is weakened by stress. However, few studies have shown how stress affects the skin except skin barrier. The objective of this study is to determine skin properties related on chronic stress.

\section{METHODS}

Three surveys confirming stress were conducted on 60 women in their age 40s. (Three surveys : Korean-translated Brief Encounter Psychosocial Instrument (BEPSI-K), Cognitive Stress Response Scale (CSRS), Stress Questionnaire for Korean National Health and Nutrition Examination Survey (KNHANES): short form) Subjects were divided into two groups with stress scores. A clinical research was conducted on 28 subjects (stress group $\mathrm{N}=14$, non-stress group $\mathrm{N}=14$ ) who agree to experimentation. The measurement parameters were hydration, skin color, elasticity, transepidermal water loss (TEWL), gloss, transparency, and wrinkles. The statistical significance was determined at $\mathrm{P}<0.05$.

\section{RESULTS/DISCUSSION}

The hydration and TEWL had no significant differences between stress group and non-stress group. But the transparency and lightness of the cheek in non-stress group were higher than stress group (Figure1. A-D) The elasticity in non-stress group was higher than stress group. But pore area in non-stress group was lower than stress group. And the data shown positive correlation between nasolabial lines and stress score (Figure2. A-C). But crow's feet, the wrinkle of middle forehead and the under the eye had no significant changes (data not shown).
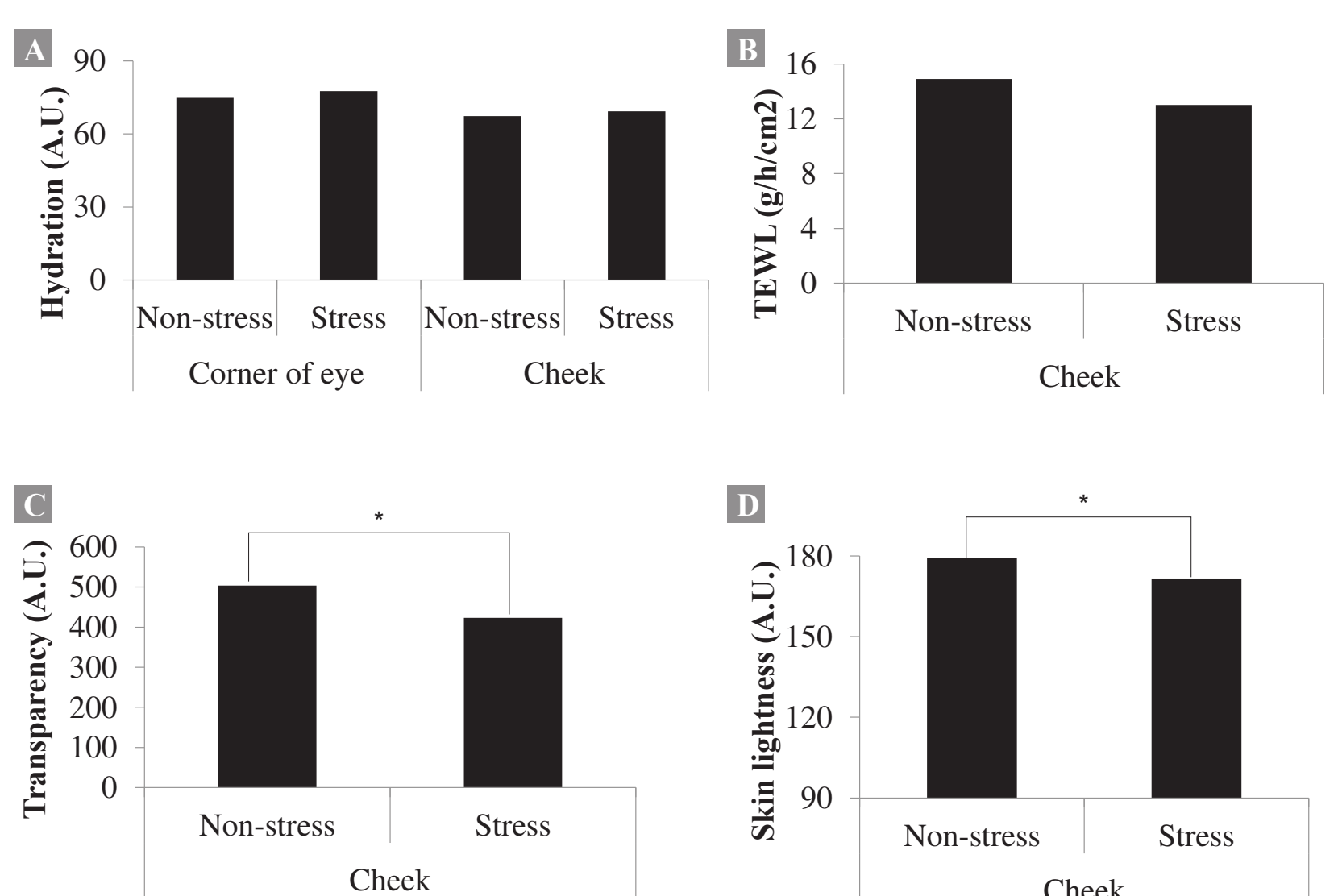

$$
\text { D }
$$

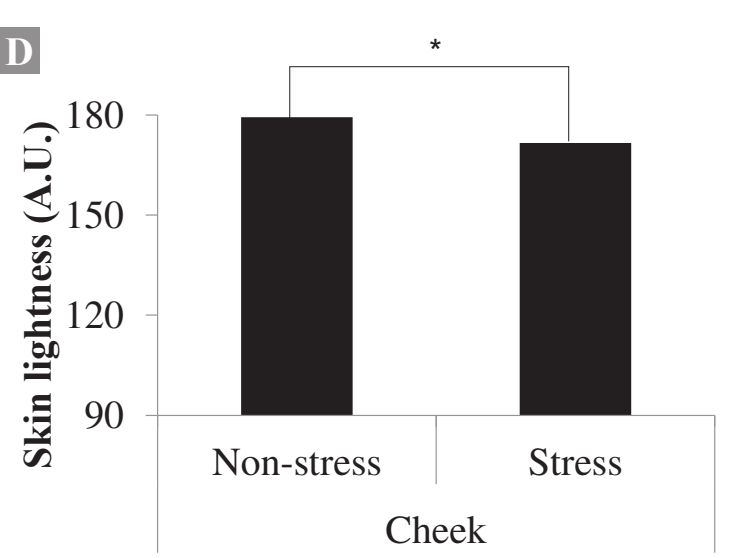

Figure 1. The Skin Barrier Function and Skin Color according to Stress The skin hydration on the cheek and the corner of eye were not statistically changed according to stress (A). The TEWL on cheek was not statistically changed according to stress (B). The cheek transparency in non-stress group was higher than stress group (C). The cheek lightness in non-stress group was higher than stress group (D).The differences were considered statistically significant at paired t-test $\left({ }^{*} p<0.05,{ }^{* *} p<0.01\right.$, $* * * p<0.001)$.

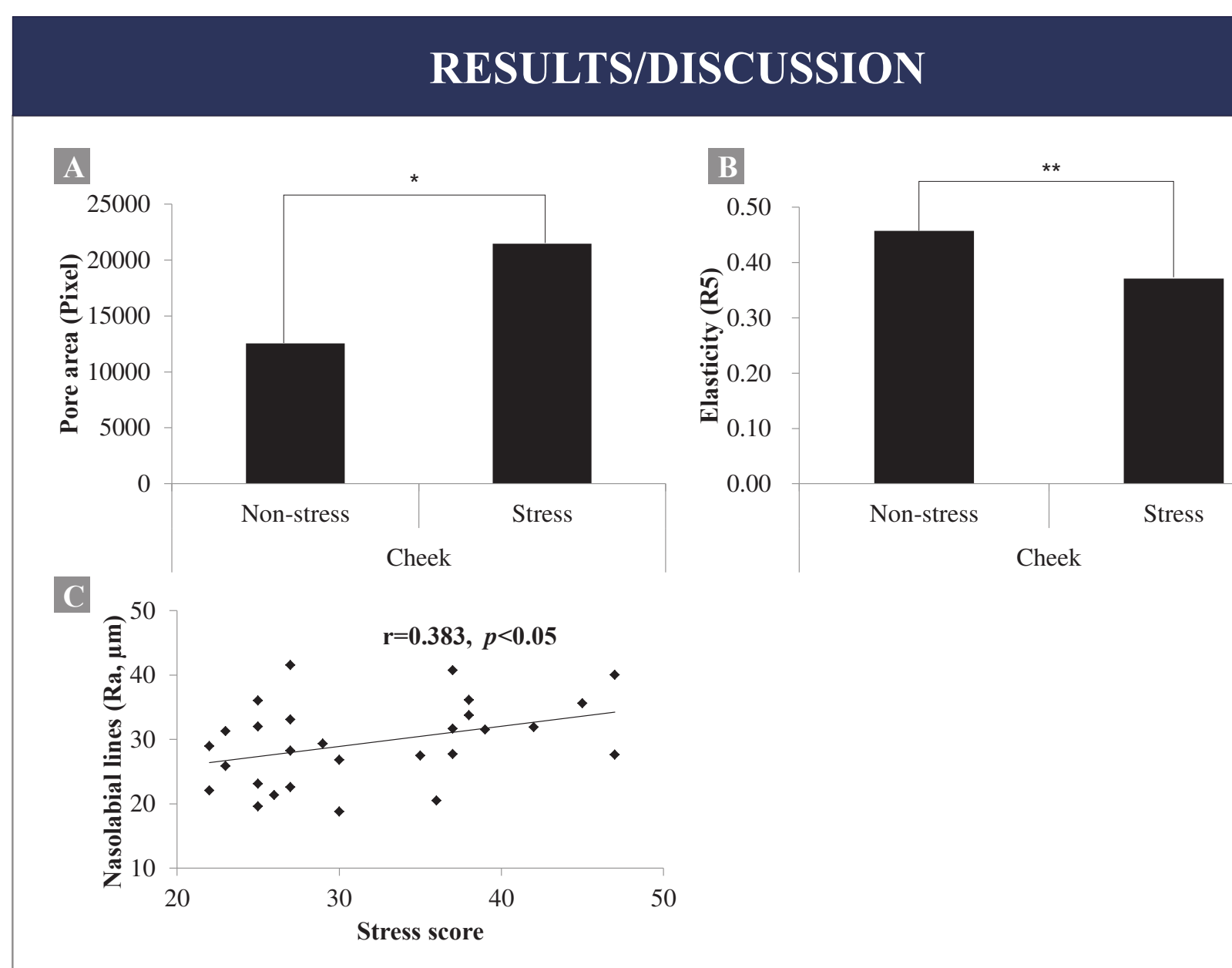

Figure 2. The Pore area, Skin Elasticity and Wrinkles according to Stress The pore area of the cheek in non-stress group was lower than stress group (A). The cheek elasticity in non-stress group was higher than stress group (B).The pearson's correlation between nasolabial lines and stress score $(\mathrm{C})$. The differences were considered statistically significant at paired t-test $(* p<0.05, * * p<0.01, * * * p<0.001)$.

We examined the beauty habits and lifestyles through the survey. The stress group responded that they have more sleep disorder than the non-stress group significantly (figure 3 . A-B).

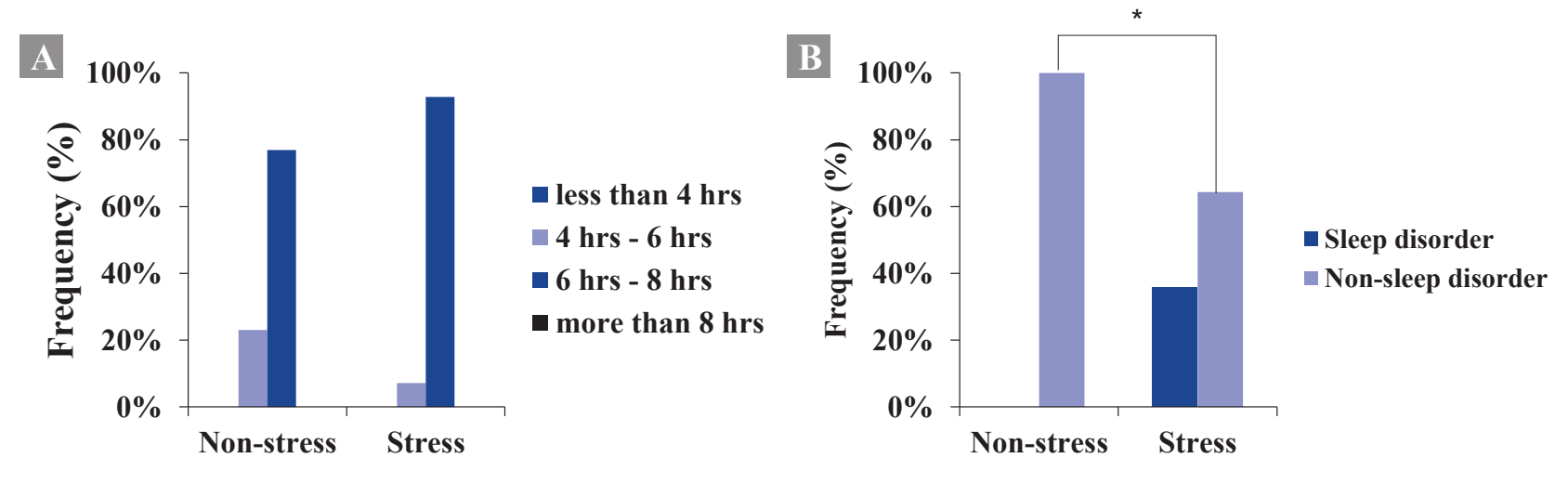

Figure 3. The Evaluation of Quantity and Quality of Sleep

The quantity of sleep was not significantly changed (A). The quality of sleep was statistically significant difference between non-stress and stress group (B). The differences were considered statistically significant at Chi-square test $(* p<0.05$, $* * p<0.01, * * * p<0.001)$

\section{CONCLUSION}

In conclusion, psychological stress affects skin color, elasticity, pores, and transparency. Also, we found that people who suffer from stress have a sleep disorder.

\section{REFERENCE}

1. A.W.M. Evers, E.W.M. Verhoeven, F.W. Kraaimaat, E.M.G.J. de Jong, S.J.M. de Brouwer, J. Schalkwijk, F.C.G.J. Sweep and P.C.M. van de Kerkhof, How stress gets under the skin: cortisol and stress reactivity in psoriasis, British Journal of Dermatology, 163 : 986-991(2010).

2. Audrey J. Henderson, Julie Lasselin, Mats Lekander, Mats J. Olsson, Simon J. Powis, John Axelsson, David I. Perrett, Skin colour changes during experimentally-induced sickness, Brain, Behavior, and Immunity 60: 312-318(2017).

3. Margaret Altemus, Babar Rao, Firdaus S. Dhabhar, Wanhong Ding, and Richard D. Granstein, Stress-Induced Changes in Skin Barrier Function in Healthy Women, $J$ Invest Dermatol 117: 309-317(2001).

4. Theodore F. Robles, Kathryn P. Brooks, and Sarah D. Pressman, Trait Positive Affect Buffers the Effects of Acute Stress on Skin Barrier Recovery, Health Psychol. 28(3): 373 378(2009). 